\title{
Cross-linking dots on metal oxides
}

\author{
Roshini Ramachandran (iD), Dahee Jung ${ }^{1}$ and Alexander M. Spokoyny (1) ${ }^{1,2}$
}

\begin{abstract}
Metal oxides are ubiquitous in our daily lives because they are robust and possess versatile electrochemical properties. Despite their popularity, these materials present limitations with respect to effective large-scale implementation. Recently, there has been growing interest in creating hybrid metal oxides to tailor the morphology and properties of these materials. From this perspective, we highlight several recent developments in cross-linked hybrid metal oxides, focusing on chemical cross-linking techniques to enrich their properties. We discuss future directions of this crosslinking approach that could enable further manipulation of these materials.
\end{abstract}

Earth-abundant metal oxides are materials whose versatile properties allow them to be utilized in applications ranging from energy storage to catalysis. ${ }^{1,2}$ Despite decades of research surrounding this class of materials, new syntheses and applications are still emerging, including novel hybrid metal oxide materials. Through nanostructuring, ${ }^{3}$ fabricating composite materials, ${ }^{4}$ or introducing new surface modifications, ${ }^{5}$ a tremendous amount of work has been done on expanding the functionality of metal oxides. In the past year alone, 1055 scientific articles related to hybrid metal oxides were published. ${ }^{6}$ Hybrid metal oxides present several notable advantages over their parent materials, one of which is the breadth of new processing options that emerge for these materials. In particular, in the case of hybrid polymer-metal oxide materials, the flexibility of the polymer is imparted to the system, which allows for facile processing into macrostructures. $^{7}$ Furthermore, the construction of hybrid metal oxides can be manipulated to fashion unique architectures on the nanoscale. Various metal oxide hybrids have been tailored with novel 2D and 3D morphologies that demonstrate superior properties (e.g., electrochemical activity) to their bulk counterparts. ${ }^{1,8}$ However, there is still room for improvement to address critical issues, such as low electrical conductivity, poor ion

\footnotetext{
Correspondence: Alexander M. Spokoyny (spokoyny@chem.ucla.edu)

'Department of Chemistry and Biochemistry, University of California, Los

Angeles, CA 90095, USA

${ }^{2}$ California NanoSystems Institute (CNSI), University of California, Los Angeles, CA 90095, USA
}

transport, and lack of homogeneity, before metal oxides can be implemented on a large scale. In this perspective article, we discuss recent scientific advances in the area of hybrid metal oxides that address these limitations.

Porosity plays a vital role in enhancing the electrochemical properties of materials; this was recently illustrated by Feng et al. in their work on mesoporous $\mathrm{Co}_{3} \mathrm{O}_{4} \cdot{ }^{9}$ Additionally, Kang et al. demonstrated that cross-linking alginate polysaccharide chains with $\mathrm{Mn}^{2+}$ ions produces a hierarchical porous $\mathrm{MnO} / \mathrm{C}$ hybrid that comprises ultrasmall $\mathrm{MnO}$ nanoparticles in a porous carbon matrix. ${ }^{10}$ This extensively cross-linked $\mathrm{MnO} / \mathrm{C}$ hybrid showed outstanding capacity and cycling performance as an electrode material for lithium-ion batteries. A similar study by Wang et al. highlighted the excellent electrochemical properties of cross-linked nanoporous $\mathrm{Co}_{3} \mathrm{O}_{4} / \mathrm{C}$ hybrids. ${ }^{11}$ Indeed, one would expect crosslinked hybrid metal oxides to exhibit a high degree of porosity due to the interconnected networks that are formed within the material. These networks enable effective charge transport by facilitating the smooth diffusion and penetration of ions (Fig. 1). It should be noted that the covalent cross-linking of inorganic-organic materials with metal oxides is superior in this regard to the physical blending of constituent materials, as in composites. For instance, Yilmaz et al. prepared highly porous $\mathrm{V}_{2} \mathrm{O}_{5}$ /graphene aerogels with thiourea as a covalent cross-linker and observed a homogenous elemental distribution in contrast to a $\mathrm{V}_{2} \mathrm{O}_{5}$ /graphene aerogel that was synthesized without the cross-linker. ${ }^{12}$ The covalently 


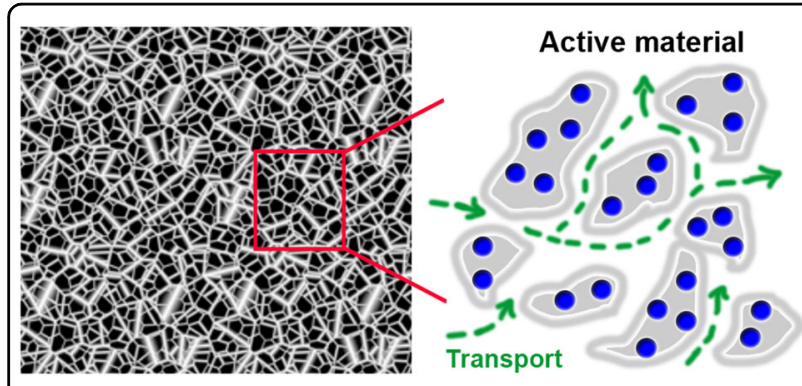

Fig. 1 Illustration of effective ion transport in porous materials by smooth diffusion and penetration of ions

cross-linked $\mathrm{V}_{2} \mathrm{O}_{5}$ /graphene aerogel displayed an enriched electrochemical performance with a high specific capacitance of $484.0 \mathrm{~F} / \mathrm{g}$ at $0.6 \mathrm{~A} / \mathrm{g}$; the noncrosslinked analog showed a specific capacitance of approximately half of that value at the same current density. ${ }^{12}$

In addition to producing a homogenous composition, covalent cross-linking offers a synergy of the constituent materials and cross-linking agent. In fact, the incorporation of sulfur in the cross-linked $\mathrm{V}_{2} \mathrm{O}_{5}$ /graphene aerogels contributed to their excellent electrochemical performance. ${ }^{12}$ Moreover, the strong chemical bonding between the individual material components (Fig. 2) reinforced the robustness and chemical stability, as opposed to physically cross-linked materials that can lose structural integrity after several electrochemical cycles. ${ }^{13,14}$ In particular, in metal oxide hybrids consisting of organic-inorganic constituents, cross-linkers ensure more efficient ion transport at the material interface, improving their electrochemical properties. ${ }^{8}$ The choice of cross-linking agent is crucial because it provides routes to generate previously unattainable functionalization for an existing metal oxide system. Several properties, such as charge separation, recombination, and transport, can be assisted by the cross-linker. For example, Chen et al. synthesized hybrid materials containing CdSe nanocrystals and $\mathrm{TiO}_{2}$ nanoparticles using mercaptopropionic acid as a bifunctional crosslinking agent. ${ }^{15}$ The resulting $\mathrm{CdSe} / \mathrm{TiO}_{2}$ heterostructure (Fig. 3a) displayed an enhanced photocatalytic hydrogen generation, which was attributed to the efficient charge separation and faster electron transfer between $\mathrm{CdSe}$ and $\mathrm{TiO}_{2}$ in the cross-linked structure. ${ }^{15}$

Titanium dioxide, one of the most recognizable metal oxides, has remained a focus of much investigation. Recently, Jung et al. reported a bottom-up approach to manipulate metal oxides through "molecular cross-linking", whereby 3D molecular boron clusters were incorporated in a $\mathrm{TiO}_{2}$ network (Fig. 3b). ${ }^{16}$ The product featured a unique structure composed of boron-rich clusters covalently cross-linked to $\mathrm{TiO}_{2}$ nanocrystals (Fig. 4). This cross-linking method not only created a
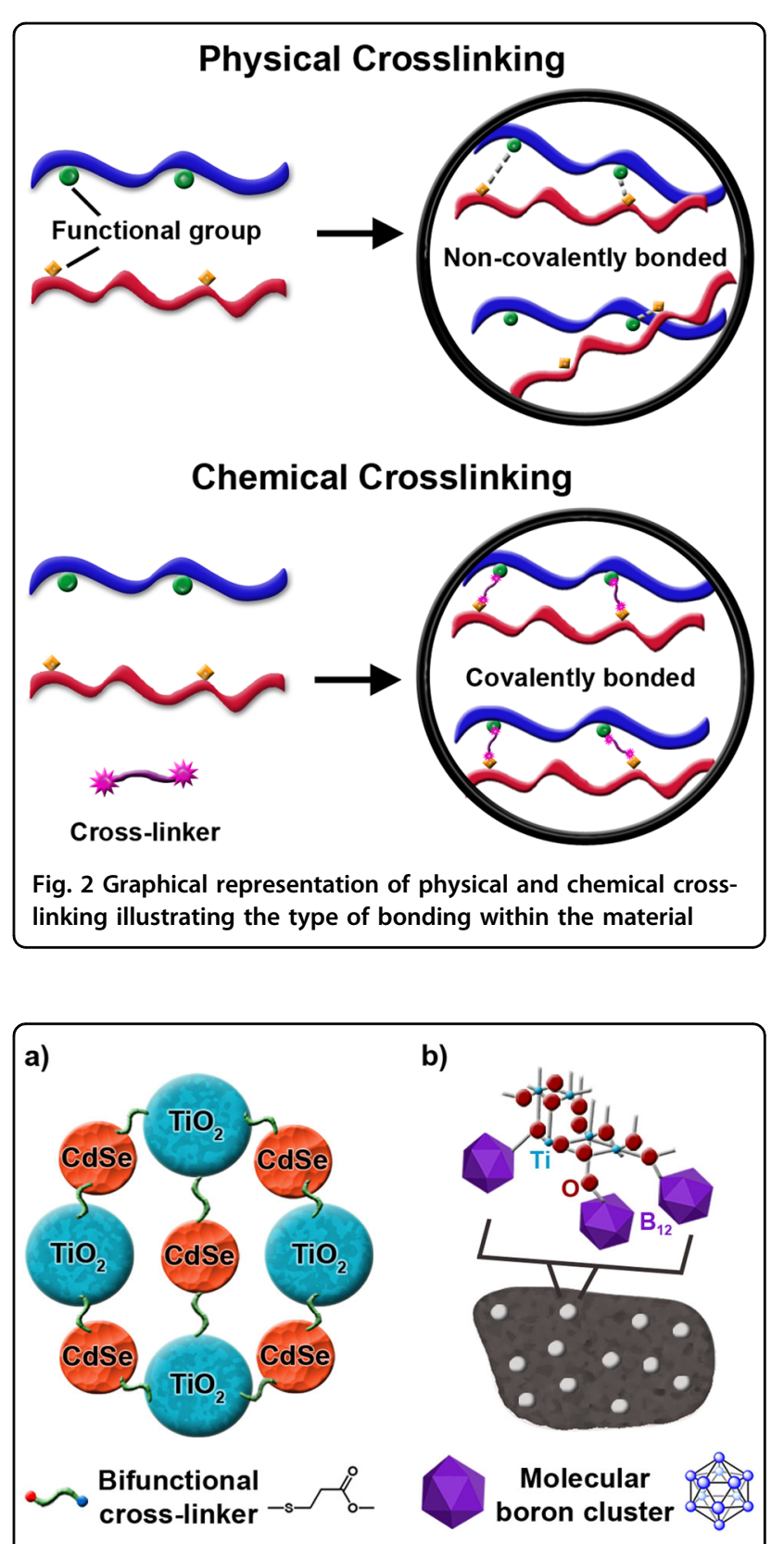

Fig. 3 Illustration of cross-linked hybrid $\mathrm{TiO}_{2}$ via. a Traditional cross-linking with an organic cross-linker and $\mathbf{b}$ molecular cross-linking utilizing boron-rich clusters

porous structure as predicted but also unexpectedly introduced an enhanced conductivity to the hybrid material. Furthermore, this new material exhibited dramatically altered light absorption properties and electrochemical behavior that was superior to pristine $\mathrm{TiO}_{2}$ merely via the introduction of the molecular boron cross-linker. ${ }^{16}$

The work by Jung et al. posed an important question: can the introduction of a cross-linking agent do more than just enrich the existing properties of a metal oxide? 

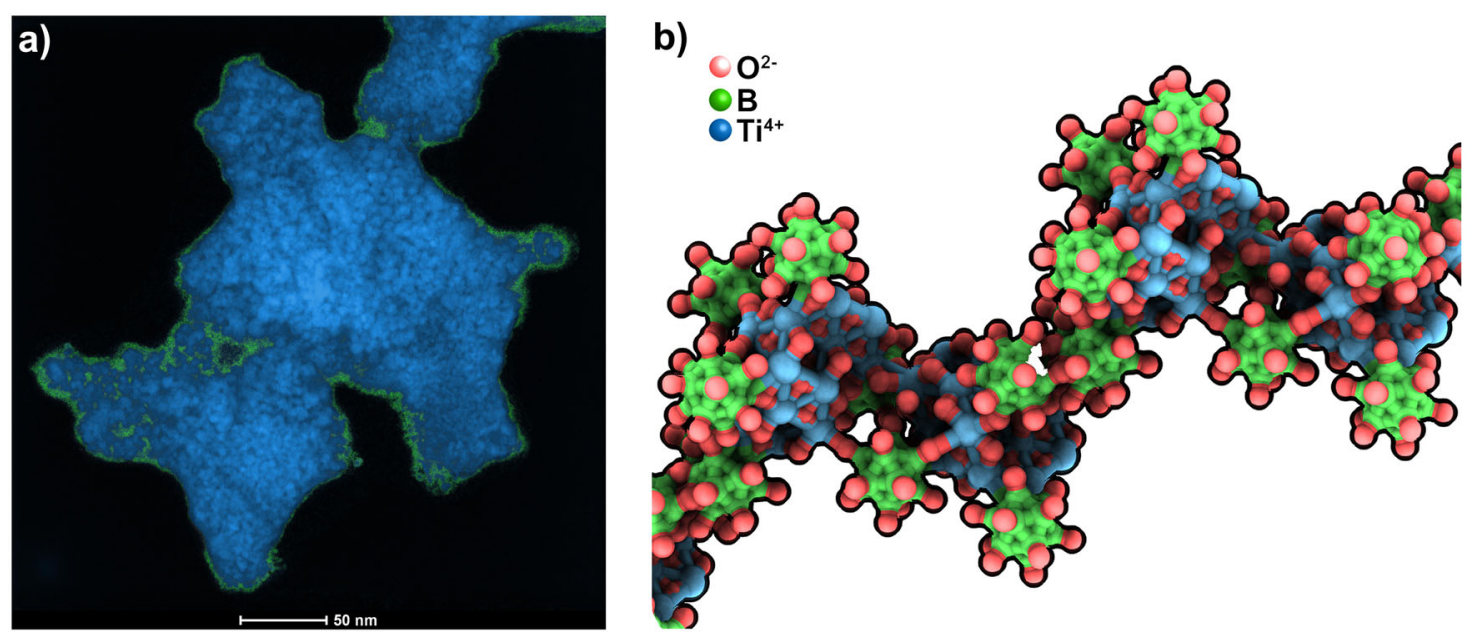

Fig. 4 Molecular cross-linked titanium dioxide hybrid material. a Scanning transmission electron microscopy (STEM) image showing $\mathrm{TiO}_{2}$ nanoparticles (blue) are densely packed in the molecular boron oxide material (green). b Simplified and nonrigorous model of the hybrid molecular boron oxide material containing $\mathrm{TiO}_{2}$ nanocrystals (adapted from ref. ${ }^{16}$ )

Table 1 Summary of recent advances in cross-linked hybrid metal oxides

\begin{tabular}{|c|c|c|c|c|c|}
\hline $\begin{array}{l}\text { Hybrid Metal } \\
\text { Oxide }\end{array}$ & Cross-linker & Morphology & Properties & Limitations & Ref. \\
\hline $\mathrm{MnO} / \mathrm{C}$ & Alginate & $\begin{array}{l}\text { Ultrasmall MnO nanoparticles in } \\
\text { porous carbon }\end{array}$ & $\begin{array}{l}\text { High specific capacity } \\
\text { Enhanced rate } \\
\text { performance } \\
\text { Long cycle life }\end{array}$ & $\begin{array}{l}\text { Aggregation of larger particles during } \\
\text { cycling } \\
\text { Capacity fading after first discharge } \\
\text { cycle }\end{array}$ & 10 \\
\hline $\mathrm{CO}_{3} \mathrm{O}_{4} / \mathrm{C}$ & Alginate & Hierarchical nanoporous structures & $\begin{array}{l}\text { High rate performance } \\
\text { Minimal capacitance loss } \\
\text { High cycling stability }\end{array}$ & $\begin{array}{l}\text { Lower specific capacitance at higher } \\
\text { carbonization temperatures due to } \\
\text { particle aggregation } \\
\text { Decreased specific capacitance at } \\
\text { increased scan rates }\end{array}$ & 11 \\
\hline $\mathrm{V}_{2} \mathrm{O}_{5} /$ graphene & Thiourea & 3-dimensional porous aerogels & $\begin{array}{l}\text { High specific capacitance } \\
\text { Good long-term cycling }\end{array}$ & $\begin{array}{l}\text { Decreased interlayer spacing of } \\
\text { graphene sheets upon annealing } \\
\text { Incomplete phase transition of } \\
\text { crystalline } \mathrm{V}_{2} \mathrm{O}_{5} \text { at } 300^{\circ} \mathrm{C}\end{array}$ & 12 \\
\hline $\mathrm{CdSe} / \mathrm{TiO}_{2}$ & $\begin{array}{l}\text { Mercaptopropionic } \\
\text { acid }\end{array}$ & $\begin{array}{l}\text { Network of CdSe quantum dot- } \\
\mathrm{TiO}_{2} \text { nanocrystal heterostructure }\end{array}$ & $\begin{array}{l}\text { Photocatalytic hydrogen } \\
\text { generation }\end{array}$ & $\begin{array}{l}\text { Decomposition of cross-linkers during } \\
\text { the long-term photocatalytic reaction }\end{array}$ & 15 \\
\hline $\mathrm{TiO}_{2} /$ boron oxide & $\begin{array}{l}\text { Perhydro-xylated } \\
\text { dodecaborate } \\
\text { clusters }\end{array}$ & $\begin{array}{l}\text { Hybrid molecular boron oxide } \\
\text { material containing embedded } \\
\mathrm{TiO}_{2} \text { nanocrystals }\end{array}$ & $\begin{array}{l}\text { Fast electron transfer rate } \\
\text { Energy storage capability } \\
\text { Photocatalytic activity } \\
\text { under visible light }\end{array}$ & $\begin{array}{l}\text { Low electrical conductivity compared to } \\
\text { other metal oxides }\end{array}$ & 16 \\
\hline
\end{tabular}

In this particular case, it was speculated that the molecular cross-linking enabled a wide range of charge transfer excitations between boron clusters in different valence states and between the clusters and the boron/titanium oxides. While the generality of this phenomenon remains to be seen, this work suggests that cross-linking can be utilized to introduce new properties to a material through appropriate choice of cross-linker.

The advantages and properties of cross-linked metal oxide hybrids from recent advances are summarized in Table 1. Future prospects of hybrid metal oxides entail the meticulous manipulation of these materials; the covalent 
cross-linking approach opens a distinct path to integrate new functionality into metal oxides as well as compensate for the shortcomings of existing metal oxide systems. Given a wide range of potential cross-linkers possessing different functionalities available, one can easily envision generating new hybrid metal oxides with diverse applications. In particular, the utilization of hyper-cross-linkers possessing multiple cross-linking sites ${ }^{17}$ can encourage a multifunctional approach towards tuning the properties of a material. This endeavor would combine practical implications along with a fundamental approach of probing these materials at the molecular level to control material properties, such as solubility and redox potential, or even impart new dimensions by employing innovative cross-linkers, such as metal complexes to generate favorable magnetic, plasmonic, or catalytic behavior. ${ }^{17-20}$ This information can guide researchers to make predictable modifications of metal oxides using well-defined cross-linkers to continually improve metal oxides. Predicting how factors influence the properties of hybrid metal oxides is paramount for shaping their tunability, especially in evaluating different metal oxides for exclusive applications.

\section{Acknowledgements}

A.M.S. is grateful to the Alfred P. Sloan Foundation for a Fellowship in chemistry, 3M for a Non-Tenured Faculty Award and the Research Corporation for Science Advancement for a Cottrell Scholar Award. The authors thank Dr. Stephan Kraemer for the STEM image and Prof. Christopher H. Hendon for the computational modeling.

\section{Conflict of interest}

UCLA has patents on the boron cluster materials discussed in this manuscript for which A.M.S. and co-workers receive royalty payments. The remaining authors declare that they have no conflict of interest.

\section{Publisher's note}

Springer Nature remains neutral with regard to jurisdictional claims in published maps and institutional affiliations.

Received: 10 October 2018 Revised: 3 February 2019 Accepted: 26 February 2019.

Published online: 19 April 2019

\section{References}

1. Jiang, J. et al. Recent advances in metal oxide-based electrode architecture design for electrochemical energy storage. Adv. Mater. 24, 5166-5180 (2012).

2. McFarland, E. W. \& Metiu, H. Catalysis by doped oxides. Chem. Rev. 113, 4391-4427 (2013).

3. Wu, H. B., Chen, J. S., Hng, H. H. \& Lou, X. W. Nanostructured metal oxide-based materials as advanced anodes for lithium-ion batteries. Nanoscale 4, 2526-2541 (2012)

4. Sreeprasad, T. S., Mailiyekkal, S. M., Lisha, K. P. \& Pradeep, T. Reduced graphene oxide-metal/metal oxide composites: facile synthesis and application in water purification. J. Hazard. Mater. 186, 921-931 (2011).

5. Chen, X., Liu., L., Yu, P. Y. \& Mao, S. S. Increasing solar absorption for photocatalysis with black hydrogenated titanium dioxide nanocrystals. Science $\mathbf{3 3 1}$ 746-750 (2011).

6. Web of Science Online, Core Collection keyword search on "hybrid metal oxide", https:/apps.webofknowledge.com, (accessed September $5^{\text {th }}$ 2018).

7. Sarkar, S., Guibal, E., Quignard, F. \& SenGupta, A. K. Polymer-supported metals and metal oxide nanoparticles: synthesis, characterization, and applications. J. Nanopart. Res. 14, 1-24 (2012).

8. Bouclé, J., Ravirajan, P. \& Nelson, J. Hybrid polymer-metal oxide thin films for photovoltaic applications. J. Mater. Chem. 17, 3141-3153 (2007).

9. Feng, D. A general ligand-assisted self-assembly approach to crystalline mesoporous metal oxides. NPG Asia Mater 10, 800-809 (2018).

10. Kang, D. et al. Crosslinking-derived $\mathrm{MnO} /$ carbon hybrid with ultrasmall nanoparticles for increasing lithium storage capacity during cycling. Carbon 99, 138-147 (2016)

11. Wang, $\mathrm{N}$. et al. Facile self-cross-linking synthesis of $3 \mathrm{D}$ nanoporous $\mathrm{CO}_{3} \mathrm{O}_{4}$ carbon hybrid electrode materials for supercapacitors. ACS Appl. Mater. Interfaces 8, 16035-16044 (2016).

12. Yilmaz, G., Lu, X. \& Ho, G. W. Cross-linker mediated formation of sulfurfunctionalized $\mathrm{V}_{2} \mathrm{O}_{5} /$ graphene aerogels and their enhanced pseudocapacitive performance. Nanoscale 9, 802-811 (2017).

13. Zeng, S. et al. Conducting polymers crosslinked with sulfur as cathode materials for high-rate, ultralong-life lithium-sulfur batteries. ChemsusChem 10, 3378-3386 (2017)

14. Wan, W. et al. Ultrafast fabrication of covalently cross-linked multifunctional graphene oxide monoliths. Adv. Funct. Mater. 24(31), 4915-4921 (2014).

15. Chuang, C. H., Qin, Z., Shen, S., Doane, T. \& Burda, C. Electron-transfer dependent photocatalytic hydrogen generation over cross-linked $\mathrm{CdSe} / \mathrm{TiO}$ type-II heterostructure. Nanotechnology 28, 084002 (2017).

16. Jung, D. et al. A molecular cross-linking approach for hybrid metal oxides. Nat Mater. 17, 341-348 (2018).

17. Zhou, N., Cao, Z. \& Xu, B. Functional hyper-crosslinkers. Chem. Eur. J. 23 15844-15851 (2017)

18. Axtell, J. C., Saleh, L. M. A., Qian, E. A., Wixtrom, A. I. \& Spokoyny, A. M. Synthesis and applications of perfunctionalized boron clusters. Inorg. Chem. 57, 2333-2350 (2018)

19. Wixtrom, A. I. et al. Tuning the electrochemical potential of perfunctionalized dodecaborate clusters through vertex differentiation. Chem. Commun. 54, 5867-5870 (2018).

20. Abb, M., Wang, Y., Papasimakis, N., De Groot, C. H. \& Muskens, O. L. Surfaceenhanced infrared spectroscopy using metal oxide plasmonic antenna arrays. Nano Lett. 14, 346-352 (2013). 